\title{
FC for Relapsed/Refractory Diffuse Large B Cell Lymphoma
}

\author{
Bingling Guo, Qiying Li*, Qingqing Huang, Xiping Liang, Chunyan Xiao, Dehong Huang, Taoyang, \\ Wenjun Zhang, Yingyu Nan, and Ying Xiang \\ Chongqing University Cancer Hospital \& Chongqing Cancer Institute \& Chongqing Cancer Hospital, China
}

*Corresponding author: Qiying Li, Chongqing University Cancer Hospital \& Chongqing Cancer Institute \& Chongqing Cancer Hospital, No.181, Hanyu Road, Shapingba district, Chongqing, China

\begin{tabular}{ll}
\hline ARTICLE INFO ABSTRACT \\
\hline
\end{tabular}

Received: 蔧 July 05, 2019

Published: 蔧 July 17, 2019

Citation: Bingling Guo, Qiying Li, Qingqing Huang, Xiping Liang, Chunyan Xiao, Dehong Huang, Taoyang, Wenjun Zhang, Yingyu Nan, Ying Xiang. FC for Relapsed/Refractory Diffuse Large B Cell Lymphoma. Biomed J Sci \& Tech Res 19(4)-2019. BJSTR. MS.ID.003344.
Keywords: FC; Relapsed/Refractory Diffuse Large B Cell Lymphoma

Abbreviations: DLBCL: Diffuse Large B-Cell Lymphoma; Auto-HCT: Autologous Hematopoietic Cell Transplantation; CR: Complete Response; PR: Partial Response; CLL: Chronic Lymphocytic Leukemia

\section{Case Report}

Despite overall improvements, relapsed/refractory diffuse large B-cell lymphoma (DLBCL)remains a major cause of morbidity [1]. Treatment option for relapsed/refractory DLBCL is salvage chemotherapy followed by an autologous hematopoietic cell transplantation(auto-HCT) for those with chemotherapy-sensitive disease [2], but prognosis is generally poor. Currently, there is no standard salvage chemotherapy regimens, and the use of new therapies for relapsed disease require further evaluation. Based on the landmark PARMA trial [2], high-dose chemotherapy and auto-HCT were firmly considered as the optimum salvage treatment in relapsed chemosensitive DLBCL. Numerous salvage chemotherapy regiments have been used, with a high response rate, low hematologic and nonhematologic toxicity. They broadly are divided into regimens based on ifosfamide, cytarabine/platinum, or gemcitabine [3], and other new drugs such as radiolabelled immunotheraphies [4].

In addition to these commonly used chemotherapy regimens, we explored some rare chemotherapy regimens. We evaluated safety and antitumor activity of the chemotherapy regimen of FC (fludarabine, cyclophosphamide) for six patients with relapsed/ refractory DLBCL. Clinical data of 6 relapsed and refractory DLBCL patients treated with FC in CHONGQING University Cancer Hospital between Feb 1.2018 and Dec 31.2018 were retrospectively analyzed. 6 patients occurred at an average age of 52.8 years. Among the 6 patients, 2 cases were transformed from follicular lymphoma and 2 from marginal lymphoma, 2 of them were relapsed DLBCL while the other 4 were refractory. There were 3 patients used rituximab combined with FC. None of them had a transplantation before the regiments. All the patients received fludarabine with $25 \mathrm{mg} / \mathrm{m} 2$ on days 1 to 3 and cyclophosphamide $300 \mathrm{mg} / \mathrm{m}^{2}$ on days 1 to 3 . After 2 to 3 cycles, we researched the ORR was $83 \%(5 / 6)$, with 2 patients achieving a complete response(CR) and 3 a partial response(PR). The major adverse events included thrombocytopenia (66.7\%), neutropenia (33.3\%), anemia (50\%) and nausea/vomiting (33.3\%), 1 patient had upper respiratory tract infection during the treatment period. There was no chemotherapy-related death occurred. The chemotherapy regimen of $\mathrm{FC}$ is effective in refractory and relapsed DLBCL, as well as safe and well-tolerated. Usually, the chemotherapy regimen of FC is used in chronic lymphocytic leukemia (CLL) and it is rarely reported in relapsed/refractory DLBCL. Our case is not large enough to perform statistical analysis, however, our findings 
explore a new possibility of traditional method. Further studies are required to practice.

\section{Conflict of Interest}

The authors declare that they have on conflict of interest.

\section{References}

1. Friedberg JW (2011) Relapsed/Refractory Diffuse Large B-Cell Lymphoma. Hematology 2011: 498-505.

\section{ISSN: 2574-1241}

DOI: $10.26717 /$ BJSTR.2019.19.003344

Qiying Li. Biomed J Sci \& Tech Res

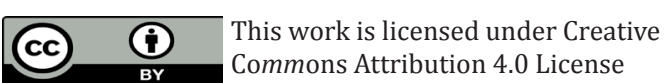

Submission Link: https://biomedres.us/submit-manuscript.php
2. Philip T, Guglielmi C, Hagenbeek A, Somers R, Van der Lelie H, et al. (1995) Autologous bone marrow transplantation as compared with salvage chemotherapy in relapses of chemotherapy-sensitive non-Hodgkin's lymphoma. New England Journal of Medicine 333(23):1540-1545.

3. Seshadri T , Kuruvilla J , Crump M (2008) Salvage Therapy for Relapsed/ Refractory Diffuse Large B Cell Lymphoma. Biology of Blood \& Marrow Transplantation 14(3):0-267.

4. Friedberg JW, Fisher RI (2008) Diffuse Large B-Cell Lymphoma. Clinical Advances in Hematology \& Oncology H \& 0 22(5): 941-952.



